Article

\title{
Biostimulating Gut Microbiome with Bilberry Anthocyanin Combo to Enhance Anti-PD-L1 Efficiency against Murine Colon Cancer
}

\author{
Xuerun Liu, Luoyang Wang *, Nan Jing $®$, Guoqiang Jiang and Zheng Liu *(i) \\ Key Lab of Industrial Biocatalysis, Ministry of Education, Department of Chemical Engineering, Tsinghua \\ University, Beijing 100084, China; liu-xr17@mails.tsinghua.edu.cn (X.L.); jn17@mails.tsinghua.edu.cn (N.J.); \\ jianggq@mail.tsinghua.edu.cn (G.J.) \\ * Correspondence: wangnan235@mail.tsinghua.edu.cn (L.W.); liuzheng@mail.tsinghua.edu.cn (Z.L.)
}

Received: 6 January 2020; Accepted: 23 January 2020; Published: 25 January 2020

check for updates

\begin{abstract}
Recent advances have revealed the essential role of gut microbiomes in the therapeutic efficiency of immune checkpoint inhibitors (ICIs). Inspired by biostimulation, a method using nutrients to accelerate the growth of soil microorganisms and the recovery of soil microbial consortia, here we propose a bilberry anthocyanin combo containing chitosan and low molecular citrus pectin (LCP), in which LCP-chitosan is used to encapsulate anthocyanins so to enhance its digestive stability and, moreover, modulate the microbiome more favorable for the PD-L1 blockade treatment. Using murine MC38 colon cancer as a model system, we examined the effects of the combo on modulating the gut microbiome and therapeutic efficiency of PD-L1 blockade treatment. It was shown that bilberry anthocyanins enriched the subdominant species, increased both the concentration and the proportion of butyrate in feces and enhanced intratumoral $\mathrm{CD} 8^{+} \mathrm{T}$ cell infiltrations. The application of the bilberry anthocyanin combo restored the species diversity of gut microbiome decreased by $\mathrm{LCP}-$ chitosan and achieved the best control of tumor growth. These preliminary results indicated unprecedented opportunities of probiotics combo in improving the therapeutic efficiency of immune checkpoint inhibitor through manipulating gut microbiome.
\end{abstract}

Keywords: immune checkpoint inhibitors; immunotherapy; gut microbiome; bilberry anthocyanins; low molecular citrus pectin; chitosan

\section{Introduction}

While immunotherapy through targeting immune checkpoints to unleash the adaptive immune response has rekindled hopes for millions of cancer patients, the response rates are below $30 \%$ for immune checkpoint inhibitors (ICIs), such as anti-programmed death 1 (PD-1) and anti-programmed death ligand 1 (PD-L1), in some tumor types [1-4]. More observations of the differences in gut microbiomes between responders and nonresponders, and, moreover, positive results in recapitulating the patient phenotype [5-7] through fecal microbiota transplant (FMT) have indicated the potential of manipulating the gut microbiome to enhance therapeutic response [7-9]. Although the underlying mechanisms remain to be discovered and differentially enriched bacterial taxa in responder patients are observed across cohorts, the significance of the gut microbiome for the therapeutic outcomes of ICIs has been recognized [10-13].

Anthocyanins are polyphenols with proven health attributes, for example, retinal protection [14]. Recently, the prebiotic function of anthocyanins has also attracted growing attention. Both in vitro fermentation of anthocyanins by human fecal microbiome [15] and in vivo dietary intervention in mice $[16,17]$ have demonstrated that anthocyanins can improve the diversity of the gut microbiome, 
promote growth of the beneficial bacterial groups while inhibit the potentially harmful bacterial groups, and increase the concentration of fecal short-chain fatty acids (SCFAs) [16,18]. All these indicate a high potential of anthocyanins in enhancing therapeutic efficiency of $\alpha$-PD-L1. However, the observation of the low recovery (below 27\%) in ileal effluent $[19,20]$ suggests the poor availability of anthocyanins in the colon and thus the need to enhance the digestive stability of anthocyanin products used for oral administration.

Biostimulation is a kind of soil remediation technique that utilizes nutrients to accelerate the growth of indigenous microorganisms to degrade contaminates [21]. An efficiency index to soil remediation is the recovery of microbial consortia that underpins the cultivation capability of soil [22]. We propose that the concept of biostimulation is also applicable for manipulating gut microbiome that is favorable for therapeutic treatment using ICIs. With appreciation of the proven effectiveness of anthocyanins in modulating the gut microbiome favorable for ICIs treatment-yet recognizing its poor digestive stability - we propose an anthocyanin combo for biostimulating the gut microbiome in order to enhance the therapeutic efficiency of the ICIs. Chitosan and low molecular citrus pectin (LCP) were added to the combo, which formed complex encapsulating anthocyanins to inhibit gastrointestinal digestion and, consequently, enhance its performance in manipulating the gut microbiome. Moreover, we consider LCP, a kind of diary fiber, as one prebiotic candidate $[23,24]$ that might also be engaged in manipulating gut microbiomes. In the present study, we examined the stimulating effects of bilberry anthocyanins and bilberry anthocyanin combo on the antitumor efficiency of the PD-L1 blockade in the mouse MC38 colon tumor model, respectively. We monitored the mice gut microbiome, fecal short-chain fatty acids and tumor $\mathrm{T}$ lymphocyte infiltration in the above-mentioned treatments to explore the underlying mechanisms of the anthocyanin combo-enhanced therapeutic efficiency of PD-L1 blockade treatment.

\section{Materials and Methods}

\subsection{Chemicals and Antibodies}

Standardized bilberry extract (MIRTOSELECT ${ }^{\circledR}$ ) was purchased from Indena S.p.A. (Milan, Italy) containing $36 \%$ of anthocyanins. The composition of the anthocyanins was determined by high-performance liquid chromatography-ultraviolet-visible spectroscopy-tandem mass spectrometry method (HPLC-UV/vis-MS/MS), as detailed below. Low molecular citrus pectin (LCP) with the molecular weight range of 5000 to 15000 Daltons was purchased from Zhejiang Gold Kropn Bio-technology Co., Ltd. (Zhejiang, China). Chitosan (C105799) was purchased from Aladdin Co. Ltd. (Shanghai, China). $\alpha$-PD-L1 monoclonal antibody (mAb) (10F.9G2) and immunoglobulin G2b (IgG2b, BE0090) isotype were purchased from BioXCell (West Lebanon, NH, USA).

\subsection{Analysis of Anthocyanins by HPLC-UV/vis-MS/MS}

HPLC-UV/vis-MS/MS analysis of anthocyanins was performed using a Shimadzu LCMS-8040 liquid chromatography with mass spectrometry (Shimadzu, Kyoto, Japan), using a TSKgel ODS-100V column $\left(5 \mu \mathrm{m}, 250 \times 4.6 \mathrm{~mm}\right.$, Tosoh Corporation, Tokyo, Japan) maintained at $35^{\circ} \mathrm{C}$. During a run, 10 $\mu \mathrm{L}$ bilberry extracts of $5 \mathrm{mg} / \mathrm{mL}$ was injected. A gradient mode of elution was performed with $1.0 \%$ $(\mathrm{v} / \mathrm{v})$ formic acid (phase A) and methanol (phase B) at a flow rate of $1 \mathrm{~mL} / \mathrm{min}$. The gradient program was set as the following: $7 \%-10 \%$ B from 0 to $2 \mathrm{~min}, 10 \%-34 \%$ B from 2 to $22.5 \mathrm{~min}$, isocratic $34 \%$ B for $2.5 \mathrm{~min}, 34 \%-70 \%$ B from 25 to $55 \mathrm{~min}, 70 \%-7 \%$ B from 55 to $60 \mathrm{~min}$, and held in isocratic $7 \% \mathrm{~B}$ for $5 \mathrm{~min}$ to clean the column. Eluents were detected at $520 \mathrm{~nm}$ (anthocyanin absorption maximum). Nitrogen was used as the nebulizing and drying gas, $3.0 \mathrm{~L} / \mathrm{min}$ and $15.0 \mathrm{~L} / \mathrm{min}$ respectively; ion spray voltage was set at $4.5 \mathrm{kV}$. Electron spray ionization mass spectra (ESI-MS) were scanned from m/z 100 to 600. Spectra were acquired in the positive ion mode for anthocyanins. Anthocyanins were identified according to their retention times and elution order in HPLC-UV/vis, followed by a comparison of their 
mass spectra in HPLC-UV/vis-MS/MS against the data in the literature [25]. Cyanidin 3-O-glucoside was further confirmed by the retention time of the available authentic standard.

\subsection{In Vitro Digestion Procedure}

Gastrointestinal digestion was conducted as previously described [26]. Firstly, we prepared 20 $\mathrm{mL}$ aqueous solution containing bilberry extracts $(1 \mathrm{mg} / \mathrm{mL})$, chitosan $(2 \mathrm{mg} / \mathrm{mL})$ and LCP $(4 \mathrm{mg} / \mathrm{mL})$, and the $\mathrm{pH}$ was adjusted to 2.0 with $\mathrm{HCl}$. Then 3144 units of pepsin were added and the sample was incubated in a $37^{\circ} \mathrm{C}$ water bath for $2 \mathrm{~h}$. Aliquots of $10 \mathrm{~mL}$ were collected and centrifuged using a Thermo Fisher Scientific Sorvall LYNX 4000 Centrifuge (Thermo Fisher Scientific, Waltham, MA, USA) at $26,000 \times g$ at $4{ }^{\circ} \mathrm{C}$ for $10 \mathrm{~min}$. The supernatant was collected for determining total anthocyanin content. The anthocyanin-LCP-chitosan complexes were also collected and washed using an $\mathrm{HCl}$ solution of $\mathrm{pH} 2.0$ three times, then the total anthocyanin content in the washing solution was determined. The $\mathrm{pH}$ of the remaining $10 \mathrm{~mL}$ from the gastric digestion step was adjusted to $\mathrm{pH} 6.8$ with $0.5 \mathrm{M} \mathrm{NaHCO}_{3}$. Afterwards, $1.25 \mathrm{~mL}$ pancreatin $(2 \mathrm{~g} / \mathrm{L}, 8 \times$ USP specifications, Sigma-Aldrich, St. Louis, MO, USA) and $1.25 \mathrm{~mL}$ of bile salts ( $25 \mathrm{~g} / \mathrm{L}$, Sigma-Aldrich, St. Louis, MO, USA) were added to each vessel and incubated for $4 \mathrm{~h}$. At $2 \mathrm{~h}$ and $4 \mathrm{~h}$, the total anthocyanin content in supernatant and anthocyanin-LCP-chitosan complexes were both determined. The total anthocyanin content of bilberry extract before and after digestion was determined by the $\mathrm{pH}$-differential spectrophotometric method [27]. Each experiment was conducted in triplicate.

\subsection{Preparation of Anthocyanin Combo}

Buffer solutions (0.2 M sodium acetate-acetic acid buffer) were prepared at $\mathrm{pH} 2.5,3.5,4.5$ and 5.5. All the chemicals including LCP, chitosan, and anthocyanins were dissolved in the buffer solution prior to use. LCP and anthocyanins mixture at a volume ratio of 1:1 were added into the chitosan solution and incubated at $4{ }^{\circ} \mathrm{C}$ for $30 \mathrm{~min}$ under constant stirring, the final concentration of chitosan, pectin and bilberry extracts was 1,2 and $0.5 \mathrm{mg} / \mathrm{mL}$, respectively. The electrostatic interaction between negatively charged LCP and positively charged chitosan may form complex encapsulating anthocyanin, thus preventing enzymatic digestion. The residual anthocyanins in the solution were recovered by centrifugation at $8000 \mathrm{rpm}$ at $4{ }^{\circ} \mathrm{C}$ for $10 \mathrm{~min}$ and determined using the $\mathrm{pH}$-differential spectrophotometric method [27]. The encapsulation efficiency (EE, \%) was calculated using the following equation:

$$
E E(\%)=\frac{\text { total amount of anthocyanins }- \text { free anthocyanins }}{\text { total amount of anthocyanins }} \times 100
$$

\subsection{Animals and Treatment}

Female C57BL/6 mice (eight weeks old) were purchased from the Vital River Laboratory Animal Technology Co. Ltd. (Beijing, China). During the experimental session, all mice were housed under pathogen-free conditions in the animal care facilities at the Institute of Biophysics, Chinese Academy of Sciences. After a one-week adaptation, mice were subcutaneously inoculated with $5 \times 10^{5} \mathrm{MC} 38$-OVA cells at the right flank. MC38 was from American Type Culture Collection (ATCC), and was cultured in 5\% $\mathrm{CO}_{2}$ and maintained in Dulbecco's Modified Eagle's Medium (Invitrogen, Carlsbad, CA, USA) supplemented with $10 \%$ heat-inactivated fetal bovine serum (HyClone, Logan, UT, USA). MC38 cells were suspended again in phosphate buffered saline (PBS) for subcutaneous injection. Tumor volumes were measured every 3-4 days with an electronic caliper and reported as volume using the formula $\left(\right.$ width $^{2} \times$ length/2). Seven days after tumor inoculation, mice were randomized by tumor size into experimental groups of 6 animals. For $\alpha$-PD-L1 treated groups, mice received twice intraperitoneal (i.p.) injection of $200 \mathrm{mg} \alpha$-PD-L1 dissolving in glucose injection at days 7 and 10. For the IgG2b isotype control, mice received isotype IgG2b at the same time point. For the $\alpha$-PD-L1/LCP-chitosan treatment group, mice were given a dose of $100 \mathrm{mg}$ pectin/ $\mathrm{kg}$ body weight and $50 \mathrm{mg}$ chitosan $/ \mathrm{kg}$ body 
weight every day. For the $\alpha$-PD-L1/anthocyanin treatment group, mice were given a dose of $25 \mathrm{mg}$ bilberry extracts/kg body weight every day. For the $\alpha$-PD-L1/anthocyanin combo treatment group, mice were given a dose of $25 \mathrm{mg}$ bilberry extracts $/ \mathrm{kg}$ body weight, $100 \mathrm{mg}$ pectin $/ \mathrm{kg}$ body weight and $50 \mathrm{mg}$ chitosan/kg body weight every day. For all groups, gavage started from day 7 following tumor inoculation. All animal procedures were performed in accordance with institutional guidelines and regulations and approved by the Institutional Animal Care and Use Committee of Institute of Biophysics, Chinese Academy of Sciences (approval ID SYXK2018-35).

\subsection{DNA Extraction and Bacterial Identification in Fecal Samples}

After 2 weeks of treatment, the feces of mice were collected and frozen at $-80{ }^{\circ} \mathrm{C}$. Bacterial DNA from the feces of mice was extracted using the QIAamp DNA Stool Mini Kit (Qiagen, Hilden, Germany) according to the manufacturer's instructions. The V3-V4 hypervariable region of $16 \mathrm{~S}$ rRNA gene was amplified with universal primers: 338F (5'-ACTCCTACGGGAGGCAGCA-3') and 806R (5'-GGACTACHVGGGTWTCTAAT-3') by PCR (ABI GeneAmp 9700, Applied Biosystems, Foster City, CA, USA) with a $20 \mu \mathrm{L}$ reaction system (TransGen, Beijing, China) containing $4 \mu \mathrm{L}$ $5 \times$ Fast Pfu Buffer, $2 \mu \mathrm{L} 2.5 \mathrm{mM}$ dNTPs, $0.8 \mu \mathrm{L}$ Forward Primer $(5 \mu \mathrm{M}), 0.8 \mu \mathrm{L}$ Reverse Primer $(5 \mu \mathrm{M}), 0.4 \mu \mathrm{L}$ Fast Pfu Polymerase, $0.2 \mu \mathrm{L}$ BSA, and $10 \mathrm{ng}$ template DNA. The procedure was as follows: $3 \mathrm{~min}$ of denaturation at $95{ }^{\circ} \mathrm{C}, 27$ cycles of $30 \mathrm{~s}$ at $95{ }^{\circ} \mathrm{C}$ (denaturation), $30 \mathrm{~s}$ for annealing at $55^{\circ}, 45 \mathrm{~s}$ at $72{ }^{\circ} \mathrm{C}$ (elongation), and a final extension at $72{ }^{\circ} \mathrm{C}$ for $10 \mathrm{~min}$. PCR products were examined by $2 \%$ agarose electrophoresis and purified using the AxyPrep DNA Gel Extraction Kit (Axygen Biosciences, Union City, CA, USA). Then, the PCR products were quantified using QuantiFluor-ST (Promega, Madison, WI, USA) and sequenced with an Illumina MiSeq platform according to the standard protocols (Shanghai Majorbio Bio-Pharm Technology Co. Ltd., Shanghai, China). The generated raw reads were screened using FASTP tool with default parameter for quality control and removal of low-quality sequences. The remaining high-quality sequences were clustered into operational taxonomic units (OTUs) at $97 \%$ of identity by using USEARCH (Version 8.1, http://www.drive5.com/usearch). Taxonomic classification was conducted using the ribosomal database project (RDP) classifier (Version 2.2, http://www.sourceforge.net/projects/rdp-classifier/) via the Silva database (Release132, http://www.arb-silva.de) with a confidence threshold of $70 \%$. Raw sequence reads of the 16S rRNA gene amplicon data are available through the SRA with accession number PRJNA598985.

\subsection{Bioinformatics Analysis}

The alpha diversity was calculated respectively for each group by package vegan and plotted with package ggplot2 in $\mathrm{R}$ (version, $\mathrm{R}$ 3.6.1). Median values and interquartile ranges are shown in boxplots. For beta-diversity (sample-to-sample dissimilarity), the principal component analysis (PCA) was performed using $\mathrm{R}$ package ade4 and illustrated with $\mathrm{R}$ package made4. The key OTUs that affect PCA clustering was extracted from result of PCA analysis and plotted with ggplot2. Enterotype analysis was performed using Calinski-Harabasz index as an indicator of optimal clustering in $\mathrm{R}$ with packages cluster, clusterSim and ade4. Linear discriminant analysis (LDA) effect size (LEfSe) were performed to investigate differences in the community composition between each enterotype (http://huttenhower.sph.harvard.edu/galaxy). PICRUSt (Phylogenetic Investigation of Communities by Reconstruction of Unobserved States) was used to evaluate the functional potential of microbial communities [28]. Briefly, BIOM format of data were constructed using QIIME against the Greengenes 13.5 database, then processed with the PICRUSt software using the EggNOG (evolutionary genealogy of genes: Non-supervised Orthologous Groups, http://eggnog.embl.de/) databases to construct the abundance tables of COGs. 


\subsection{Quantification of Fecal Short-Chain Fatty Acids (SCFAs) by Gas Chromatography}

Feces samples of $0.1 \mathrm{~g}$ were suspended in $0.4 \mathrm{~mL}$ water and $0.1 \mathrm{~mL} 50 \%$ sulphuric acid, homogenized for $5 \mathrm{~min}$ and centrifuged at 13,000 $\mathrm{g}$ for $10 \mathrm{~min}$. The supernatant was extracted with ethyl ether of equal volume and the suspension was obtained for gas chromatography on Shimadzu GC-2010 system (Shimadzu, Kyoto, Japan) equipped with a flame ionization detector (FID). Separation was achieved using a HP-INNOWax column $(30 \mathrm{~m} \times 0.250 \mathrm{~mm} \times 0.25 \mu \mathrm{m}$, Agilent Technologies Inc., Santa Clara, CA, USA). Split ratio was 10:1, the pressure of carrier gas, helium, was maintained at $100 \mathrm{kPa}$. Injection volume was $1 \mu \mathrm{L}$. Helium $(30 \mathrm{~mL} / \mathrm{min})$, hydrogen $(40 \mathrm{~mL} / \mathrm{min})$ and dry air $(400 \mathrm{~mL} / \mathrm{min})$ were used as auxiliary gases for the flame ionization detector. The injector and detector temperatures were $250{ }^{\circ} \mathrm{C}$ and $280^{\circ} \mathrm{C}$, respectively. The oven temperature was held at $60^{\circ} \mathrm{C}$ at first, then increased to $100^{\circ} \mathrm{C}$ at a rate of $20^{\circ} \mathrm{C} / \mathrm{min}$, and maintained for $3 \mathrm{~min}$, finally to $210^{\circ} \mathrm{C}$ at a rate of $30^{\circ} \mathrm{C} / \mathrm{min}$ and maintained for $5 \mathrm{~min}$.

\subsection{Quantification of Immune Cells in Tumor Tissues by Flow Cytometry}

Tumors were excised on day 14 after inoculation. The isolated tumor tissues were digested with Collagenase IV (Yeasen Biotech Co., Ltd., Shanghai, China) at $37^{\circ} \mathrm{C}$ for $2 \mathrm{~h}$, and then filtered through a 70- $\mu \mathrm{m}$ cell strainer (Corning Incorporated, Corning, NY, USA) to obtain the single cells. The harvested cells were washed twice with PBS, and then stained in the dark with anti-mouse antibodies for CD4 (GK1.5), CD8 (53-6.7), CD45 (30-F11) (BioLegend, San Diego, CA, USA) at $4{ }^{\circ} \mathrm{C}$ for $30 \mathrm{~min}$. Analysis was performed immediately by using an FACS Calibur flow cytometer (Becton-Dickinson, Fullerton, CA, USA).

\subsection{Statistical Analysis}

The statistical analysis was performed by GraphPad Prism 8 with two-tailed Student's $t$-test or repeated measures ANOVA (time $\times$ tumor volume) with Sidak's multiple comparisons test. All data were presented as mean \pm SD. $p<0.05$ were considered to be statistically remarkable, while $p<0.01$ indicate that the differences reach the levels of statistical high significance.

\section{Results}

\subsection{The Protection Effects of Chitosan/Pectin for Anthocyanins Against Gastrointestinal Digestion}

Parent ions, and daughter ions of detected anthocyanins are summarized in Table 1. Bilberry anthocyanins contain 20 major monomeric anthocyanins, including five anthocyanidins (delphinidin, cyanidin, petunidin, peonidin and malvidin) and their monoglycoside derivatives [29]. When incubated in simulated gastric fluid without pepsin, the total content of both anthocyanins and polyphenols remained constant throughout $2 \mathrm{~h}$ (Table S1), and even kept stable when $\mathrm{pH}$ raised up to 5.5 (Figure S1). In the case of simulated intestinal fluid with pancreatin, the total content of anthocyanins and polyphenols decreased to $47.3 \%$ and $91.5 \%$, respectively, after $4 \mathrm{~h}$ incubation (Figure S2), suggesting the poor stability of anthocyanins in the small intestine. As shown in Figure 1B, chitosan and LCP forms polyelectrolyte complexes at different $\mathrm{pHs}(2.5,3.5,4.5$ and 5.5) encapsulating anthocyanins, in which the maximum encapsulation efficiency of $47 \%$ is achieved at $\mathrm{pH} 3.5$. It is noted that chitosan is soluble in simulated gastric fluid (SGF) but dissoluble in simulated intestinal fluid (SIF). It is anticipated that chitosan will form precipitate encapsulating anthocyanins when the $\mathrm{pH}$ increases from simulated gastric fluid to simulated intestinal fluid. Indeed, our experiment demonstrated that chitosan could enhance the digestive stability of anthocyanins (Figure S3). Similarly, the encapsulation by LCP-chitosan also leads to an improved stability of anthocyanins in simulated gastrointestinal fluid (Figure 1C). All these suggest an effective protection of anthocyanins from the gastrointestinal digestion, which is favorable for the delivery of anthocyanin to the colon. 
Table 1. Peak identification of anthocyanins in Mirtoselect ${ }^{\circledR}$ bilberry extract using HPLC-UV/vis-MS/MS.

\begin{tabular}{|c|c|c|c|c|}
\hline Peak \# & Species & Formula & $\begin{array}{l}\text { Molecular Ions } \\
(\mathrm{m} / \mathrm{z})\end{array}$ & $\begin{array}{l}\text { Fragment Ions } \\
\operatorname{MS}^{2}(\mathrm{~m} / \mathrm{z})\end{array}$ \\
\hline 1 & Delphinidin-3-O-galactoside & $\mathrm{C}_{21} \mathrm{H}_{21} \mathrm{O}_{12}$ & 465 & 303 \\
\hline 2 & Delphinidin-3-O-glucoside & $\mathrm{C}_{21} \mathrm{H}_{21} \mathrm{O}_{12}$ & 465 & 303 \\
\hline 3 & Cyanidin-3-O-galactoside & $\mathrm{C}_{21} \mathrm{H}_{21} \mathrm{O}_{11}$ & 449 & 287 \\
\hline 4 & Delphinidin-3-O-arabinoside & $\mathrm{C}_{20} \mathrm{H}_{19} \mathrm{O}_{11}$ & 435 & 303 \\
\hline 5 & Cyanidin-3-O-glucoside & $\mathrm{C}_{21} \mathrm{H}_{21} \mathrm{O}_{11}$ & 449 & 287 \\
\hline 6 & Petunidin-3-O-galactoside & $\mathrm{C}_{22} \mathrm{H}_{23} \mathrm{O}_{12}$ & 479 & 317 \\
\hline 7 & $\begin{array}{l}\text { Petunidin-3-O-glucoside/ } \\
\text { Cyanidin-3-O-arabinoside }\end{array}$ & $\begin{array}{l}\mathrm{C}_{22} \mathrm{H}_{23} \mathrm{O}_{12} / \\
\mathrm{C}_{20} \mathrm{H}_{19} \mathrm{O}_{10}\end{array}$ & $479 / 419$ & $317 / 287$ \\
\hline 8 & Peonidin-3-O-galactoside & $\mathrm{C}_{22} \mathrm{H}_{23} \mathrm{O}_{11}$ & 463 & 301 \\
\hline 9 & $\begin{array}{l}\text { Petunidin-3-O-arabinoside/ } \\
\text { Peonidin-3-O-glucoside/ } \\
\text { Malvidin-3-O-galactoside }\end{array}$ & $\begin{array}{l}\mathrm{C}_{21} \mathrm{H}_{21} \mathrm{O}_{11} / \\
\mathrm{C}_{22} \mathrm{H}_{23} \mathrm{O}_{11} / \\
\mathrm{C}_{23} \mathrm{H}_{25} \mathrm{O}_{12}\end{array}$ & $449 / 463 / 493$ & $317 / 301 / 331$ \\
\hline 10 & Malvidin-3-O-glucoside & $\mathrm{C}_{23} \mathrm{H}_{25} \mathrm{O}_{12}$ & 493 & 331 \\
\hline 11 & Peonidin-3-arabinoside & $\mathrm{C}_{21} \mathrm{H}_{21} \mathrm{O}_{10}$ & 433 & 301 \\
\hline 12 & Malvidin-3-O-arabinoside & $\mathrm{C}_{22} \mathrm{H}_{23} \mathrm{O}_{11}$ & 463 & 331 \\
\hline 13 & delphinidin & $\mathrm{C}_{15} \mathrm{H}_{11} \mathrm{O}_{7}$ & 303 & 303,229 \\
\hline 14 & Cyanidin & $\mathrm{C}_{15} \mathrm{H}_{11} \mathrm{O}_{6}$ & 287 & 287,137 \\
\hline 15 & Petunidin & $\mathrm{C}_{16} \mathrm{H}_{13} \mathrm{O}_{7}$ & 317 & 203,245 \\
\hline 16 & Peonidin & $\mathrm{C}_{16} \mathrm{H}_{13} \mathrm{O}_{6}$ & 301 & 201,229 \\
\hline 17 & Malvidin & $\mathrm{C}_{17} \mathrm{H}_{15} \mathrm{O}_{7}$ & 331 & $315,242,287$ \\
\hline
\end{tabular}
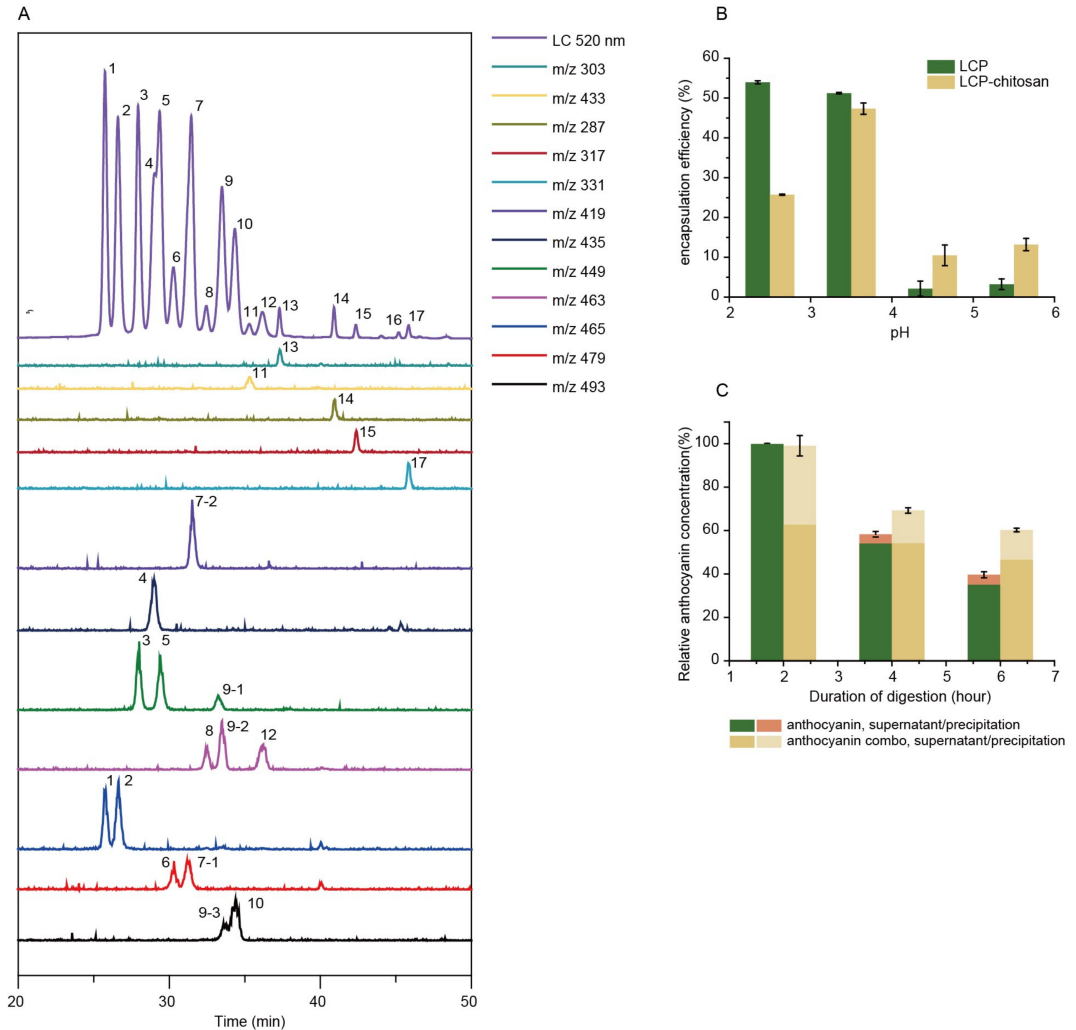

Figure 1. Main components and digest stability

(A) High-performance liquid chromatography-ultraviolet-visible spectroscopy-tandem mass spectrometry (HPLC-UV/vis-MS/MS) chromatograms (detected at $520 \mathrm{~nm}$ ) of bilberry extracts. (B) Chitosan and low molecular citrus pectin (LCP) fabricated polyelectrolyte complexes at different pHs. (C) Relative content of anthocyanins of bilberry extracts and anthocyanin combo (anthocyanin-LCP-chitosan) in simulated gastric fluid (SGF) with pepsin at $\mathrm{pH} 2.0$ for $2 \mathrm{~h}(\mathrm{~A})$ and subsequently in simulated intestinal fluid (SIF) with pancreatin at $\mathrm{pH} 6.8$ for $4 \mathrm{~h}$. 


\subsection{Effects of Anthocyanin and Anthocyanin Combo on the Anti-PD-L1 Efficiency in Mouse MC38 Colon Tumor}

Here we examined the effects of anthocyanin, anthocyanin combo on the $\alpha$-PD-L1 treatment. It is anticipated that the encapsulation by LCP-chitosan may deliver more anthocyanin to colon and thus offers an intensified enhancement. Meanwhile LCP, which could induce SCFA formation [23] and promote the growth of beneficial gut bacteria [24], may also impact on the gut microbiome and thus the $\alpha$-PD-L1 treatment. As illustrated in Figure 2A, twice intraperitoneal injection (i.p.) of $\alpha$-PD-L1 $\mathrm{mAb}$ on the 7th and 10th day after tumor inoculation caused tumor growth delay compared to the control group. Oral supplement with anthocyanins could improve the therapeutic effects of $\alpha$-PD-L1 $\mathrm{mAb}$. Moreover, the application of the anthocyanin combo further increased therapeutic effectivity of $\alpha$-PD-L1 mAb. In comparison, solely supplement with LCP-chitosan achieved no obvious benefits.

A

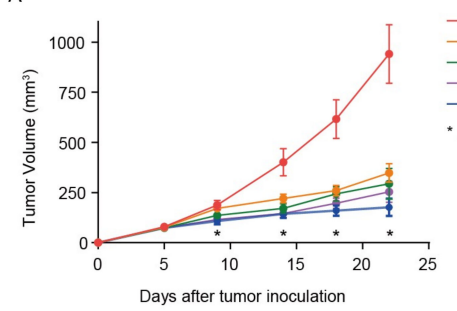

C

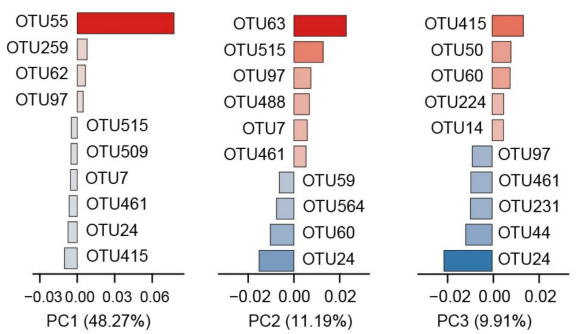

$E$

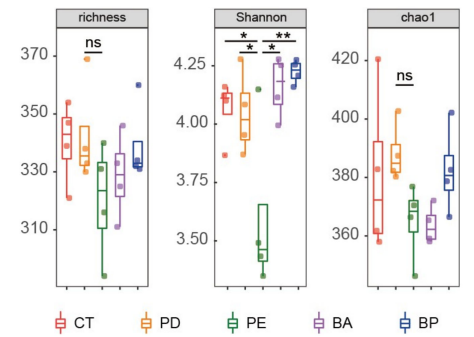

$\because \mathrm{CT}$, control $-\mathrm{PD}, \alpha-P D-L 1$
$\mathrm{PE}, \alpha-P D-L 1 / L C P-c h i t o s a n$ BA, $\alpha-P D-L 1 / a n t h o c y a n i n$ $P<0.05$, Repeated measures ANOVA
B

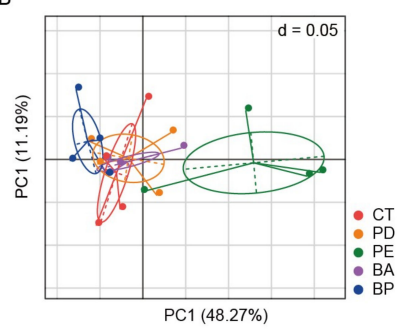

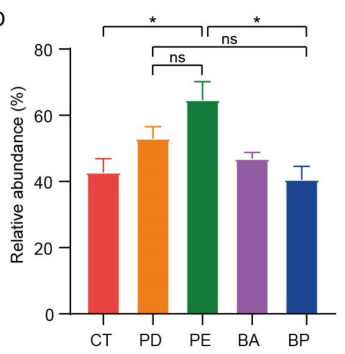

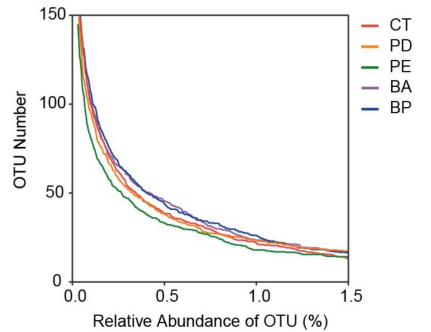

Figure 2. Oral administration of bilberry anthocyanins enhances anti-PD-L1 efficiency and changes the mouse gut microbiome structure. (A) C57BL/6 mice were inoculated subcutaneously with $5 \times$ $10^{5}$ MC38-OVA cells and treated with $200 \mu \mathrm{g}$ of the immunoglobulin G2b (IgG2b) isotype or the $\alpha$-PD-L1 mAb on days 7 and 10. CT, the IgG2b isotype control; PD, the $\alpha$-PD-L1 group; PE, the $\alpha$-PD-L1/LCP-chitosan group; BA, the $\alpha$-PD-L1/anthocyanin group; BP, the $\alpha$-PD-L1/anthocyanin combo group. Repeated measures ANOVA (time $\times$ tumor volume) and Sidak's multiple comparisons test were used to test mouse tumor growth between groups. Data indicates mean \pm SEM, $n=6$ per group, ${ }^{*} p<0.05$. (B) Beta-diversity analysis of microbial communities by using principal component analysis (PCA) based on OTUs. (C) The associated contribution plot illustrating the key OTUs that impact the PCA clustering. (D) Significant increase of Muribaculaceae in the $\alpha$-PD-L1/LCP-chitosan group. (E) For alpha diversity, richness, chao1, Shannon, Simpson, and ace indices were calculated using random subsamples of 35,043 sequences per sample, Data indicates mean \pm SEM. ${ }^{*} p<0.05$, ** $p<0.01$ (Student's $t$-test). (F) OTU richness under different thresholds of relative abundance. 


\subsection{Diversity of the Gut Microbiome of Mice with Different Treatment}

Mice feces samples in above study were collected for microbial diversity analysis of 16S rRNA after 2 weeks of treatment. Firstly, we performed analysis of beta diversity at OUT level. As shown in Figure 2B, the principal component analysis (PCA) showed significant changes (Table S2, Adonis variance analysis based on Bray-Curtis distance matrices at OTU level) in the composition of gut microbiome after oral supplement with LCP-chitosan and anthocyanin combo. There were 5 key OTUs that affect the PCA clustering, i.e., OTU55, OTU63, OTU24, OTU415, OTU515 (Figure 2C). OTU55 belonging to Muribaculaceae was significantly enriched in the $\alpha$-PD-L1/LCP-chitosan group $(p=0.04342$, Kruskal-Wallis test), while OTU415 affiliated to Lachnospiraceae was enriched in the anthocyanin combo group. Further analyses indicate an enrichment of Muribaculaceae in the $\alpha$-PD-L1/LCP-chitosan group (Figure 2D), which is the most abundant commensal at the family level.

Higher species diversity is correlated with higher response rates to ICIs [30,31], hence we analyzed the alpha diversity. As shown in Figure 2E, compared with the control group or the $\alpha$-PD-L1 group, the alpha diversity indices of the communities from samples of the $\alpha$-PD-L1/LCP-chitosan group were significantly decreased, indicating a profound impact of LCP-chitosan on mice gut microbiome. Interestingly, the alpha diversity indices of samples from anthocyanin combo group returned to the level of control group, hinting a synergy effects between anthocyanins, LCP and chitosan. Further analysis shows that LCP-chitosan obviously decease the subdominant species diversity, while bilberry anthocyanins enrich the subdominant species and recover LCP-chitosan induced decrease in these species (Figure 2F).

\subsection{Analysis of Differential Taxons}

We then compared the community structure of each group at different taxonomic levels. As shown in Figure 3A,B, at the phylum level, the Firmicutes to Bacteroidetes ratio $(\mathrm{F} / \mathrm{B})$ decreased in the $\alpha$-PD-L1 group compared to the control group, and further decreased when combining with LCP-chitosan, while increased when combining with bilberry anthocyanins though there are no significant differences between each of them. Besides, the F/B ratio in $\alpha$-PD-L1/anthocyanin combo group returned to the level of control group, hinting at possible improvement in energy harvest from food for hosts [32]. At the family level, Muribaculaceae and Lachnospiraceae were the most dominant commensals in the gut microbiome across all groups (Figure 3C). Compared with the $\alpha$-PD-L1 group, the abundance of Lachnospiraceae increased in the $\alpha$-PD-L1/anthocyanin group and the $\alpha$-PD-L1/anthocyanin combo group while the abundance of Muribaculaceae decreased.

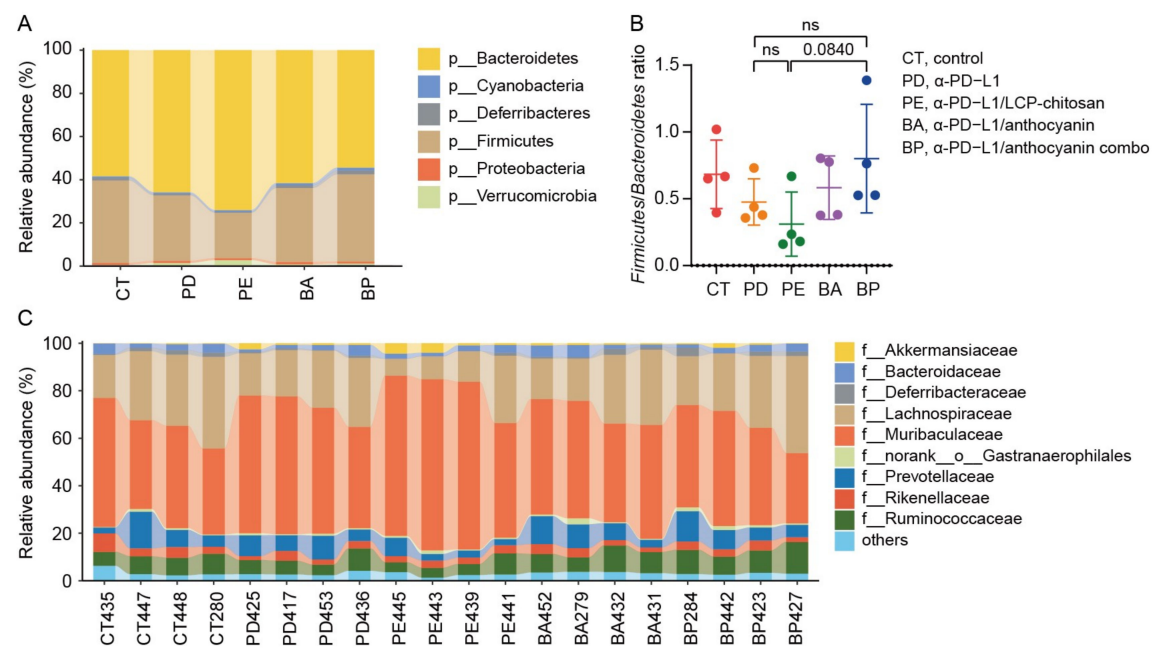

Figure 3. Composition of the gut microbiome at different taxonomic levels. (A) Phylum level taxonomic composition of bacterial communities in each group. (B) The Firmicutes to Bacteroidetes ratio. (C) Taxonomic composition of the bacterial communities in each group at family level. 


\subsection{Enterotype Analysis and Functional Differences in the Gut Microbiome}

All the samples can be clustered into three groups using the Jensen-Shannon Distance (JSD) at the OTUs level (Figure 4A). Enterotype 1 was mainly from the $\alpha$-PD-L1/anthocyanin group and the $\alpha$-PD-L1/anthocyanin combo group, enterotype 2 was from the control group and the $\alpha$-PD-L1 group, enterotype 3 from the $\alpha$-PD-L1/LCP-chitosan group. LEfSe (Linear discriminant analysis Effect Size) analysis showed differential gut microbiome signatures between these three enterotypes (Figure 4B). Ruminococcaceae and Lachnospiraceae were enriched in enterotype1, while Muribaculaceae was enriched in enterotype3. Subsequently, we performed the analysis of 16S-predicted functional profiles for studying the information behind the differences of the gut microbiome structure among clusters. As shown in Figure $4 \mathrm{C}$, some gene functions show significant differences, among which carbohydrate transport and metabolism was significantly higher in enterotype1 and enterotype2 compared to enterotype3 (Figure 4D). Since one of the major factors shaping the gut microbiome is the influx of dietary fiber into the intestine [33], and the major metabolites of dietary fiber, such as SCFAs, are important for maintaining host health [34], increasing in carbohydrate transport and metabolism in enterotype1 and enterotype 2 hints possible improvement in energy metabolism (Figure 4D). Besides, there were significant differences in the F/B ratio between three enterotypes ( $p=0.0076$, Kruskal-Wallis test), ratio of F/B in enteroype1 and enteroype2 were significantly higher than that in enterotype3 (Figure 4E), further revealing that anthocyanins could change energy metabolism of the gut microbiome, which may account for the changes in the structure and diversity of the gut microbiome induced by oral supplement with anthocyanins.

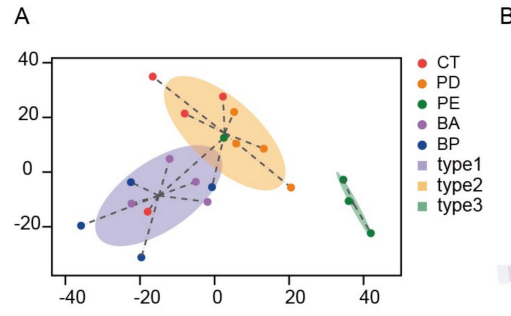

C

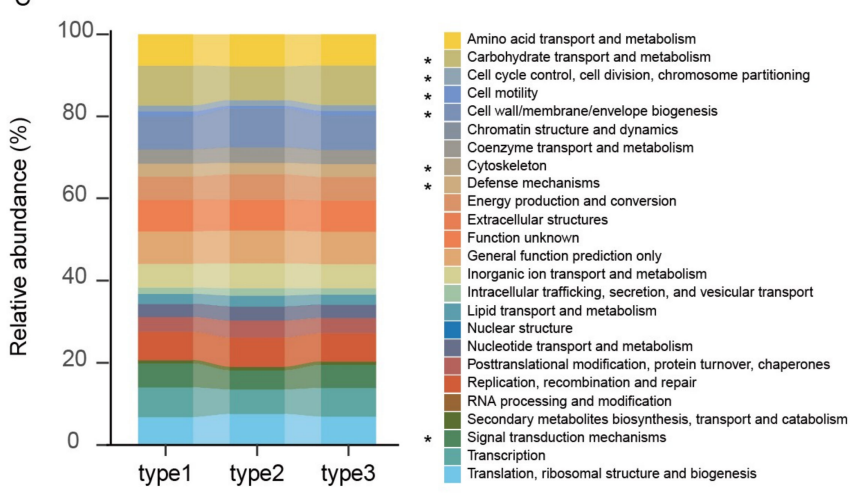

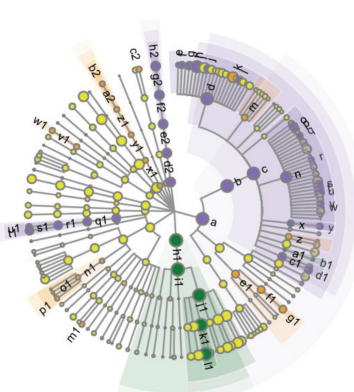

$=$ type1 $=$ type2 $=$ type2
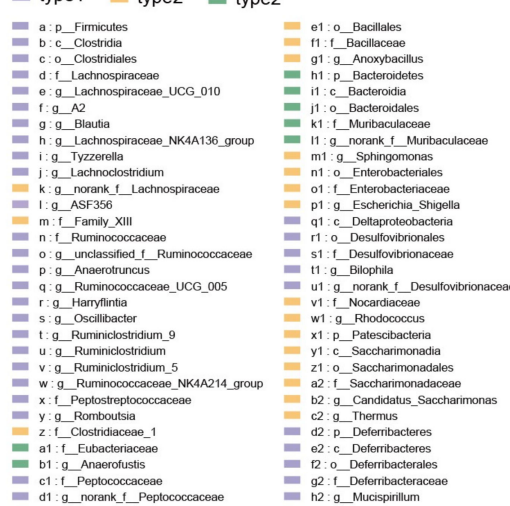

D

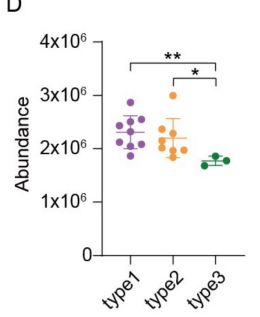

E

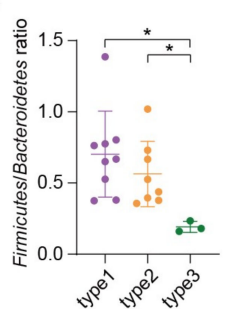

Figure 4. Enterotype analysis and functional differences between different enterotypes. (A) Gut microbiome was clustered into three enterotypes at OTU-level using Jensen-Shannon distance, and the graphs for enterotype clusters indicate the first two principal coordinates. (B) Linear discriminant analysis effect size (LEfSe) was used to differentiate the three enterotypes. (C) PICRUSt prediction of functional profiling of the microbial communities from these three enterotypes based on the 16SrRNA gene sequences. (D) Difference in the function of carbohydrate transport and metabolism and (E) The Firmicutes to Bacteroidetes ratio were tested using Student's $t$-test, ${ }^{*} p<0.05,{ }^{* *} p<0.01$. 


\subsection{Oral Supplement with Anthocyanin and Anthocyanin Combo Changes the Production of Fecal SCFAs}

Microbial fermentation of nondigestible dietary carbohydrates (NDCs) mainly leads to the production of SCFAs, among which propionate and butyrate in particular exert a wide range of health-promoting functions [35]. Thus, we also collected mice feces for SCFAs assay. As illustrated in Figure 5A, the total content of SCFAs was significantly reduced in the $\alpha$-PD-L1 group in contrast with the control group ( $p=0.0474$, unpaired $t$-test with Welch's correction), which was partially recovered when PD-L1 blockade was combined with LCP-chitosan or bilberry anthocyanins. Furthermore, oral supplement with bilberry anthocyanins increased both the concentration and the proportion of butyrate (Figure $5 \mathrm{C}-\mathrm{H}$ ).
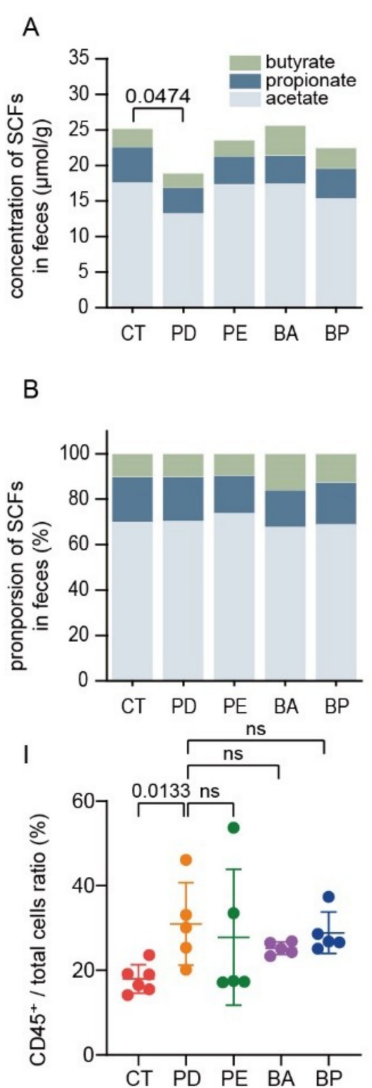
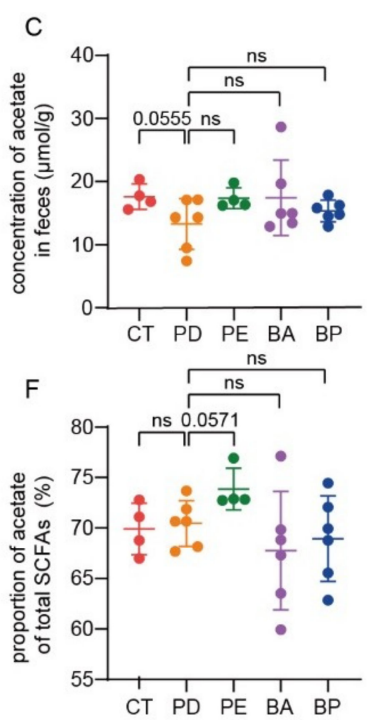

$\mathrm{J}$

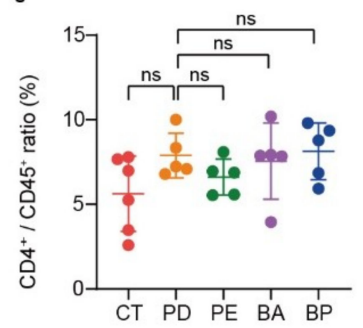

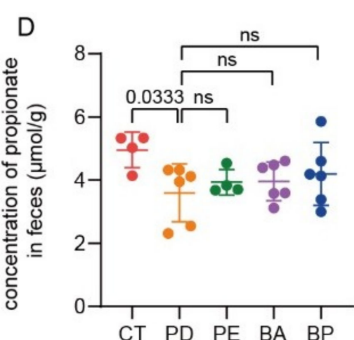
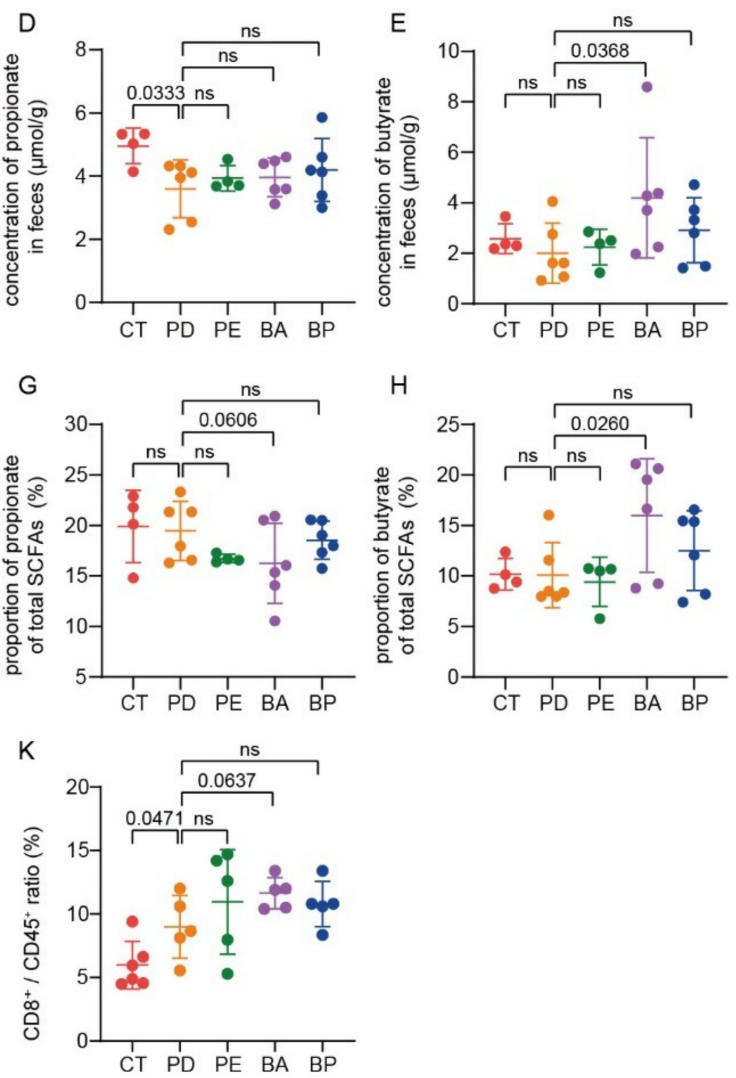

Figure 5. Bilberry anthocyanins promote the production of short-chain fatty acids (SCFAs) and tumor immune infiltration. The total content of SCFAs (A) and the proportions of individual to total SCFAs in feces. (B) Quantification of the content of acetate (C), propionate (D) and butyrate (E) in feces by gas chromatography. The proportions of acetate $(\mathbf{F})$, propionate $(\mathbf{G})$ and butyrate $(\mathbf{H})$ to total SCFAs in feces. Quantification of total T lymphocytes $(\mathbf{I}), \mathrm{CD}^{+}(\mathbf{J})$ and $\mathrm{CD}^{+}(\mathbf{K}) \mathrm{T}$ cells in tumor tissues by flow cytometry. Differences were tested using Student's $t$-test; ns, not significant.

\subsection{Bilberry Anthocyanins Enhance Tumor Immune Infiltration}

Finally, the percentage of $\mathrm{CD}^{+}$or $\mathrm{CD}^{+} \mathrm{T}$ cells in tumor tissues was analyzed by flow cytometry. As detailed in Figure 5I, the proportion of lymphocytes to total cells was increased in all $\alpha$-PD-L1 treatment groups, indicating enhanced tumor immune infiltration. Besides, there were smaller intragroup differences in the proportion of lymphocytes to total cells in the $\alpha$-PD-L1/anthocyanin group and the $\alpha$-PD-L1/anthocyanin combo group, which was consistent with the observation that these two groups had a smaller intragroup difference in tumor volume (Figure S4). Meanwhile, compared with the PD-L1 blockade alone, treatment with PD-L1 blockade combined with anthocyanins or anthocyanin combo showed a higher proportion of $\mathrm{CD}^{+} \mathrm{T}$ cells to total lymphocytes from tumor tissues (Figure $5 \mathrm{~K}$ ), which was associated with better control of tumor growth. 


\section{Discussion}

The discovery that gut microbiome influences the clinical outcomes of ICIs has attracted immense efforts on manipulating the gut microbiome to enhance therapeutic response [10]. On the other hand, great efforts are needed to explore the interaction between microbiome and ICIs. For example, there are reports that the obese patients had a better outcome with ICIs [36-39]. Different gut microbiome signatures were observed between obese people and lean people [40] and the gut microbiome had been involved in the onset of obesity-related disorders [41]. Dietary anthocyanins have been found a role against obesity and inflammation [42] and in metabolic changes [43]. Therefore, the elucidation of the role of anthocyanins in enhancing ICIs efficiency, as observed in the present study, should be put into the context of both the metabolism and immune system. In this study, we found that oral supplement with anthocyanins or anthocyanin combo enriched the subdominant bacteria, and the PD-L1/anthocyanin combo group achieved the highest efficiency in the $\alpha$-PD-L1 treatments. The addition of anthocyanins in the $\alpha$-PD-L1 treatment showed an overrepresentation of Lachnospiraceae and Ruminococcaceae, and a higher F/B ratio. Lachnospiraceae and Ruminococcaceae belong to the order Clostridiales within the class Clostridia of the phylum Firmicutes, and many members of this family are butyrate-producing species [35,44]. These structure changes in the gut microbiome contributed to the increased production of SCFAs in anthocyanins treatment groups, especially butyrate. Butyrate is mainly metabolized by colonic cells, and it exerts anticarcinogenic and anti-inflammatory effects, and can improve intestinal mucosal barrier function [45]. Therefore, SCFAs produced by Lachnospiraceae and Ruminococcaceae may play a critical role in enhancing the $\alpha$-PD-L1 mAb efficiency by oral supplement with bilberry anthocyanins.

It is worthy to note that routinely used agents may interfere with gut microbiome and thus the response to ICIs. For example, proton pump inhibitors (PPIs) used for potent gastric acid suppression have been found to perturb the gut microbiome, leading to dysbiosis and decreased efficiency of treatment with ICIs [46-48]. More recently, Kim et al. reported that antibiotics are associated with poor progression-free survival (PFS) and overall survival (OS) in patients with solid cancers treated with ICIs [49]. The present study shown that LCP-chitosan significantly increased the relative abundance of the Muribaculaceae, one of the most abundant families in the gut microbiome, while decreased the species diversity. We note that the efficiency of PD-L1 blockade treatment was not obviously influenced in comparison with the $\alpha$-PD-L1 group, indicating that there are other gut microbiome signatures contributing to the efficiency of PD-L1 blockade treatment, in addition to the species diversity. The application of anthocyanin combo restored the distorted gut microbiome caused by LCP-chitosan, and, in the meantime, achieved the highest therapeutic efficiency of ICIs. The elucidation of the further improvement of ICIs efficiency by the anthocyanin combo, as compared to the anthocyanins treatment that also yielded an increased concentration of butyrate and enhanced tumor immune infiltration, needs additional investigation. Collectively, these findings demonstrate again the connection between gut microbiome with the therapeutic efficiency of ICIs and, more importantly, indicate unprecedented opportunities to manipulate gut microbiome favorable for ICIs with natural nutrients, e.g., probiotics, as stimulants.

\section{Conclusions}

In the present study, we proposed an idea in terms of biostimulation of the gut microbiome, that is, using natural nutrients to manipulate the gut microbiome so to enhance the therapeutic efficiency of PD-L1 blockade. We demonstrated this idea using anthocyanins and anthocyanin combo containing chitosan and LCP, respectively. It was shown that the oral administration of bilberry anthocyanins showed an overrepresentation of Lachnospiraceae and Ruminococcaceae in which many members are butyrate-producing species, which was consistent with the increased concentration and proportion of butyrate. The bilberry anthocyanins could change the energy metabolism of the gut microbiome, which may account for the regulation of the gut microbiome. Oral supplementation with bilberry anthocyanins during the PD-L1 blockade treatment showed enhanced tumor immune infiltration, which 
was associated with better control of tumor growth. The stimulating effects of bilberry anthocyanins were further intensified via using an anthocyanin combo. Although the underpinning mechanisms of enhanced tumor immune infiltration and the antitumor efficiency of the PD-L1 blockade by anthocyanin and anthocyanin combo remain to be discovered, our results reconfirmed the connection of gut microbiome with ICIs and revealed the opportunities of biostimulation of gut microbiome in checkpoint inhibitor therapy.

Supplementary Materials: The following are available online at http://www.mdpi.com/2076-2607/8/2/175/s1.

Author Contributions: Formal analysis, X.L.; Funding acquisition, G.J. and Z.L.; Investigation, X.L. and N.J.; Methodology, L.W.; Validation, L.W., G.J. and Z.L.; Writing-original draft, X.L.; Writing-review \& editing, L.W., G.J. and Z.L. All the authors discussed the results and commented on the manuscript. All authors have read and agreed to the published version of the manuscript.

Funding: This work was funded by Xinjiang Tianhui Information Technology Co. Ltd. and Xinjiang Tianjianhemu Biotech Co. Ltd. under project contract No. 20192000168 and No. 20172000941.

Conflicts of Interest: The authors declare no conflicts of interest.

\section{References}

1. Borghaei, H.; Paz-Ares, L.; Horn, L.; Spigel, D.R.; Steins, M.; Ready, N.E.; Chow, L.Q.; Vokes, E.E.; Felip, E.; Holgado, E.; et al. Nivolumab versus docetaxel in advanced nonsquamous non-small-cell lung cancer. $N$. Engl. J. Med. 2015, 373, 1627-1639. [CrossRef]

2. Motzer, R.J.; Escudier, B.; McDermott, D.F.; George, S.; Hammers, H.J.; Srinivas, S.; Tykodi, S.S.; Sosman, J.A.; Procopio, G.; Plimack, E.R.; et al. Nivolumab versus everolimus in advanced renal-cell carcinoma. N. Engl. J. Med. 2015, 373, 1803-1813. [CrossRef] [PubMed]

3. Ribas, A.; Wolchok, J.D. Cancer immunotherapy using checkpoint blockade. Science 2018, 359, $1350-1355$. [CrossRef] [PubMed]

4. Yang, J.C.; Hughes, M.; Kammula, U.; Royal, R.; Sherry, R.M.; Topalian, S.L.; Suri, K.B.; Levy, C.; Allen, T.; Mavroukakis, S.; et al. Ipilimumab (anti-CTLA4 antibody) causes regression of metastatic renal cell cancer associated with enteritis and hypophysitis. J. Immunother. 2007, 30, 825-830. [CrossRef] [PubMed]

5. Gopalakrishnan, V.; Spencer, C.N.; Nezi, L.; Reuben, A.; Andrews, M.C.; Karpinets, T.V.; Prieto, P.A.; Vicente, D.; Hoffman, K.; Wei, S.C.; et al. Gut microbiome modulates response to anti-PD-1 immunotherapy in melanoma patients. Science 2018, 359, 97-103. [CrossRef]

6. Matson, V.; Fessler, J.; Bao, R.; Chongsuwat, T.; Zha, Y.Y.; Alegre, M.L.; Luke, J.J.; Gajewski, T.F. The commensal microbiome is associated with anti-PD-1 efficacy in metastatic melanoma patients. Science 2018, 359, 104-108. [CrossRef]

7. Routy, B.; Le Chatelier, E.; Derosa, L.; Duong, C.P.M.; Alou, M.T.; Daillere, R.; Fluckiger, A.; Messaoudene, M.; Rauber, C.; Roberti, M.P.; et al. Gut microbiome influences efficacy of PD-1-based immunotherapy against epithelial tumors. Science 2018, 359, 91-97. [CrossRef]

8. Sivan, A.; Corrales, L.; Hubert, N.; Williams, J.B.; Aquino-Michaels, K.; Earley, Z.M.; Benyamin, F.W.; Lei, Y.M.; Jabri, B.; Alegre, M.L.; et al. Commensal Bifidobacterium promotes antitumor immunity and facilitates anti-PD-L1 efficacy. Science 2015, 350, 1084-1089. [CrossRef]

9. Vetizou, M.; Pitt, J.M.; Daillere, R.; Lepage, P.; Waldschmitt, N.; Flament, C.; Rusakiewicz, S.; Routy, B.; Roberti, M.P.; Duong, C.P.M.; et al. Anticancer immunotherapy by CTLA-4 blockade relies on the gut microbiota. Science 2015, 350, 1079-1084. [CrossRef]

10. Helmink, B.A.; Khan, M.A.W.; Hermann, A.; Gopalakrishnan, V.; Wargo, J.A. The microbiome, cancer, and cancer therapy. Nat. Med. 2019, 25, 377-388. [CrossRef]

11. Jobin, C. Precision medicine using microbiota. Science 2018, 359, 32-34. [CrossRef] [PubMed]

12. Faria, A.; Fernandes, I.; Norberto, S.; Mateus, N.; Calhau, C. Interplay between anthocyanins and gut microbiota. J. Agric. Food Chem. 2014, 62, 6898-6902. [CrossRef] [PubMed]

13. Igwe, E.O.; Charlton, K.E.; Probst, Y.C.; Kent, K.; Netzel, M.E. A systematic literature review of the effect of anthocyanins on gut microbiota populations. J. Hum. Nutr. Diet 2019, 32, 53-62. [CrossRef] [PubMed]

14. Castañeda-Ovando, A.; Pacheco-Hernández, M.d.L.; Páez-Hernández, M.E.; Rodríguez, J.A.; Galán-Vidal, C.A. Chemical studies of anthocyanins: A review. Food Chem. 2009, 113, 859-871. [CrossRef] 
15. Hidalgo, M.; Oruna-Concha, M.J.; Kolida, S.; Walton, G.E.; Kallithraka, S.; Spencer, J.P.; de Pascual-Teresa, S. Metabolism of anthocyanins by human gut microflora and their influence on gut bacterial growth. J. Agric. Food Chem. 2012, 60, 3882-3890. [CrossRef]

16. Li, J.; Wu, T.; Li, N.; Wang, X.N.; Chen, G.Y.; Lyu, X.L. Bilberry anthocyanin extract promotes intestinal barrier function and inhibits digestive enzyme activity by regulating the gut microbiota in aging rats. Food Funct. 2019, 10, 333-343. [CrossRef]

17. Pan, P.; Lam, V.; Salzman, N.; Huang, Y.W.; Yu, J.; Zhang, J.; Wang, L.S. Black raspberries and their anthocyanin and fiber fractions alter the composition and diversity of gut microbiota in F-344 rats. Nutr. Cancer 2017, 69, 943-951. [CrossRef]

18. Garcia-Mazcorro, J.F.; Lage, N.N.; Mertens-Talcott, S.; Talcott, S.; Chew, B.; Dowd, S.E.; Kawas, J.R.; Noratto, G.D. Effect of dark sweet cherry powder consumption on the gut microbiota, short-chain fatty acids, and biomarkers of gut health in obese $\mathrm{db} / \mathrm{db}$ mice. Peer] 2018, 6, e4195. [CrossRef]

19. McDougall, G.J.; Conner, S.; Pereira-Caro, G.; Gonzalez-Barrio, R.; Brown, E.M.; Verrall, S.; Stewart, D.; Moffet, T.; Ibars, M.; Lawther, R.; et al. Tracking (poly)phenol components from raspberries in ileal fluid. J. Agric. Food Chem. 2014, 62, 7631-7641. [CrossRef]

20. Stalmach, A.; Edwards, C.A.; Wightman, J.D.; Crozier, A. Gastrointestinal stability and bioavailability of (poly)phenolic compounds following ingestion of Concord grape juice by humans. Mol. Nutr. Food Res. 2012, 56, 497-509. [CrossRef]

21. Seklemova, E.; Pavlova, A.; Kovacheva, K. Biostimulation-based bioremediation of diesel fuel: Field demonstration. Biodegradation 2001, 12, 311-316. [CrossRef] [PubMed]

22. Dong, Y.; Lang, Z.; Kong, X.; Lu, D.; Liu, Z. Kinetic and multidimensional profiling of accelerated degradation of oil sludge by biostimulation. Env. Sci. Process. Impacts 2015, 17, 763-774. [CrossRef] [PubMed]

23. Bindels, L.B.; Delzenne, N.M.; Cani, P.D.; Walter, J. Towards a more comprehensive concept for prebiotics. Nat. Rev. Gastroenterol. Hepatol. 2015, 12, 303-310. [CrossRef] [PubMed]

24. Ferreira-Lazarte, A.; Kachrimanidou, V.; Villamiel, M.; Rastall, R.A.; Moreno, F.J. In vitro fermentation properties of pectins and enzymatic-modified pectins obtained from different renewable bioresources. Carbohydr. Polym. 2018, 199, 482-491. [CrossRef]

25. Barnes, J.S.; Nguyen, H.P.; Shen, S.; Schug, K.A. General method for extraction of blueberry anthocyanins and identification using high performance liquid chromatography-electrospray ionization-ion trap-time of flight-mass spectrometry. J. Chromatogr. A 2009, 1216, 4728-4735. [CrossRef]

26. Correa-Betanzo, J.; Allen-Vercoe, E.; McDonald, J.; Schroeter, K.; Corredig, M.; Paliyath, G. Stability and biological activity of wild blueberry (Vaccinium angustifolium) polyphenols during simulated in vitro gastrointestinal digestion. Food Chem. 2014, 165, 522-531. [CrossRef]

27. AOAC. Total monomeric anthocyanin pigment content of fruit juices, beverages, natural colorants and wine. J. AOAC Int. 2005, 88, 1269.

28. Langille, M.G.; Zaneveld, J.; Caporaso, J.G.; McDonald, D.; Knights, D.; Reyes, J.A.; Clemente, J.C.; Burkepile, D.E.; Vega Thurber, R.L.; Knight, R.; et al. Predictive functional profiling of microbial communities using 16S rRNA marker gene sequences. Nat. Biotechnol. 2013, 31, 814-821. [CrossRef]

29. Cassinese, C.; de Combarieu, E.; Falzoni, M.; Fuzzati, N.; Pace, R.; Sardone, N. New liquid chromatography method with ultraviolet detection for analysis of anthocyanins and anthocyanidins in Vaccinium myrtillus fruit dry extracts and commercial preparations. J. AOAC Int. 2007, 90, 911-919. [CrossRef]

30. Derosa, L.; Hellmann, M.D.; Spaziano, M.; Halpenny, D.; Fidelle, M.; Rizvi, H.; Long, N.; Plodkowski, A.J.; Arbour, K.C.; Chaft, J.E.; et al. Negative association of antibiotics on clinical activity of immune checkpoint inhibitors in patients with advanced renal cell and non-small-cell lung cancer. Ann. Oncol. 2018, 29, 1437-1444. [CrossRef]

31. Jin, Y.; Dong, H.; Xia, L.; Yang, Y.; Zhu, Y.; Shen, Y.; Zheng, H.; Yao, C.; Wang, Y.; Lu, S. The diversity of gut microbiome is associated with favorable responses to anti-programmed death 1 immunotherapy in Chinese patients with NSCLC. J. Thorac. Oncol. 2019, 14, 1378-1389. [CrossRef] [PubMed]

32. Vaiserman, A.M.; Koliada, A.K.; Marotta, F. Gut microbiota: A player in aging and a target for anti-aging intervention. Ageing Res. Rev. 2017, 35, 36-45. [CrossRef] [PubMed]

33. Koropatkin, N.M.; Cameron, E.A.; Martens, E.C. How glycan metabolism shapes the human gut microbiota. Nat. Rev. Microbiol. 2012, 10, 323-335. [CrossRef] [PubMed] 
34. Cockburn, D.W.; Koropatkin, N.M. Polysaccharide degradation by the intestinal microbiota and its influence on human health and disease. J. Mol. Biol. 2016, 428, 3230-3252. [CrossRef] [PubMed]

35. Louis, P.; Flint, H.J. Formation of propionate and butyrate by the human colonic microbiota. Environ. Microbiol. 2017, 19, 29-41. [CrossRef] [PubMed]

36. Cortellini, A.; Bersanelli, M.; Buti, S.; Cannita, K.; Santini, D.; Perrone, F.; Giusti, R.; Tiseo, M.; Michiara, M.; Di Marino, P. A multicenter study of body mass index in cancer patients treated with anti-PD-1/PD-L1 immune checkpoint inhibitors: When overweight becomes favorable. J. Immunother. Cancer 2019, 7, 57. [CrossRef]

37. Xu, H.; Cao, D.; He, A.; Ge, W. The prognostic role of obesity is independent of sex in cancer patients treated with immune checkpoint inhibitors: A pooled analysis of 4090 cancer patients. Int. Immunopharmacol. 2019, 74, 105745. [CrossRef]

38. Ichihara, E.; Harada, D.; Inoue, K.; Sato, K.; Hosokawa, S.; Kishino, D.; Watanabe, K.; Ochi, N.; Oda, N.; Hara, N. The impact of body mass index on the efficacy of anti-PD-1/PD-L1 antibodies in patients with non-small cell lung cancer. Lung Cancer 2020, 139, 140-145. [CrossRef]

39. Kichenadasse, G.; Miners, J.O.; Mangoni, A.A.; Rowland, A.; Hopkins, A.M.; Sorich, M.J. Association between body mass index and overall survival with immune checkpoint inhibitor therapy for advanced non-small cell lung cancer. JAMA Oncol. 2019. [CrossRef]

40. Ley, R.E.; Turnbaugh, P.J.; Klein, S.; Gordon, J.I. Microbial ecology: Human gut microbes associated with obesity. Nature 2006, 444, 1022. [CrossRef]

41. Cani, P.D.; Van Hul, M.; Lefort, C.; Depommier, C.; Rastelli, M.; Everard, A. Microbial regulation of organismal energy homeostasis. Nat. Metab. 2019, 1, 34. [CrossRef]

42. Lee, Y.-M.; Yoon, Y.; Yoon, H.; Park, H.-M.; Song, S.; Yeum, K.-J. Dietary anthocyanins against obesity and inflammation. Nutrients 2017, 9, 1089. [CrossRef] [PubMed]

43. Overall, J.; Bonney, S.; Wilson, M.; Beermann, A.; Grace, M.; Esposito, D.; Lila, M.; Komarnytsky, S. Metabolic effects of berries with structurally diverse anthocyanins. Int. J. Mol. Sci. 2017, 18, 422. [CrossRef]

44. Meehan, C.J.; Beiko, R.G. A phylogenomic view of ecological specialization in the Lachnospiraceae, a family of digestive tract-associated bacteria. Genome Biol. Evol. 2014, 6, 703-713. [CrossRef] [PubMed]

45. Reichardt, N.; Vollmer, M.; Holtrop, G.; Farquharson, F.M.; Wefers, D.; Bunzel, M.; Duncan, S.H.; Drew, J.E.; Williams, L.M.; Milligan, G.; et al. Specific substrate-driven changes in human faecal microbiota composition contrast with functional redundancy in short-chain fatty acid production. ISME J. 2018, 12, 610-622. [CrossRef] [PubMed]

46. Homicsko, K.; Richtig, G.; Tuchmann, F.; Tsourti, Z.; Hanahan, D.; Coukos, G.; Wind-Rotolo, M.; Richtig, E.; Zygoura, P.; Holler, C. Proton pump inhibitors negatively impact survival of PD-1 inhibitor based therapies in metastatic melanoma patients. Ann. Oncol. 2018, 29, mdy511.001. [CrossRef]

47. Trabolsi, A.; Winter, M.; Rodriguez, E. Proton pump inhibitors and response to immune check-point inhibitors: Single center study. Am. Soc. Clin. Oncol. 2019, 37, e14092. [CrossRef]

48. Mukherjee, S.; Ibrahimi, S.; Khalid, B.; Roman, D.; Zhao, D.; Aljumaily, R. Do proton pump inhibitors modulate the efficacy of anti-PD-1/PD-L1 therapy? A retrospective study. J. Oncol. Pharm. Pract. 2019, 25, 762-764. [CrossRef]

49. Kim, H.; Lee, J.E.; Hong, S.H.; Lee, M.A.; Kang, J.H.; Kim, I.-H. The effect of antibiotics on the clinical outcomes of patients with solid cancers undergoing immune checkpoint inhibitor treatment: A retrospective study. BMC Cancer 2019, 19, 1100. [CrossRef]

(C) 2020 by the authors. Licensee MDPI, Basel, Switzerland. This article is an open access article distributed under the terms and conditions of the Creative Commons Attribution (CC BY) license (http://creativecommons.org/licenses/by/4.0/). 